\title{
The prior statistics of object colors
}

\author{
Jan J. Koenderink \\ Delft University of Technology, Faculty of Electrical Engineering, Mathematics, and Computer Science, Mekelweg 4, \\ 2628 CD Delft, The Netherlands (j.j.koenderink@tudelft.nl)
}

Received March 25, 2009; revised October 25, 2009; accepted October 27, 2009; posted December 11, 2009 (Doc. ID 109249); published January 19, 2010

\begin{abstract}
The prior statistics of object colors is of much interest because extensive statistical investigations of reflectance spectra reveal highly non-uniform structure in color space common to several very different databases. This common structure is due to the visual system rather than to the statistics of environmental structure. Analysis involves an investigation of the proper sample space of spectral reflectance factors and of the statistical consequences of the projection of spectral reflectances on the color solid. Even in the case of reflectance statistics that are translationally invariant with respect to the wavelength dimension, the statistics of object colors is highly non-uniform. The qualitative nature of this non-uniformity is due to trichromacy. (C) 2010 Optical Society of America

OCIS codes: $330.1690,330.1710,330.1715,330.1720,330.1730,330.6180$.
\end{abstract}

\section{INTRODUCTION}

Various speculations [1-3] as to the nature of human color vision require the prior statistics of object colors for the generic human biotope (or perhaps certain prehistorical, savannah, or rain forest primate biotopes). Unfortunately, the space of the spectra is huge, the effective dimensionality (formally, the dimension is infinite) being in the 10100 range [4]. Existing databases necessarily highly undersample these riches and also are necessarily highly biased toward limited classes of spectra, either by design or by accident. Moreover, various uncertainties typically render results suspect to various degrees (e.g., reflectances near zero or unity are largely useless [5]). Recent attempts at the empirical determination of such prior statistics [6-8] reveal non-trivial non-uniformities. The heroic attempt by Philipona and O'Regan [8] is especially interesting, as it reveals highly specific structure. A close examination of these data suggests that the clustering occurs in the directions from which the Goethe edge color loci (see below) approach the black and white vertices of the Schrödinger color solid. If this were the case, then the non-uniformities would be due to the structure of the visual system (in the final instance the photopigment action spectra) rather than to ecological factors. This again would introduce a vicious circle in many of the aforementioned speculative reasonings. Thus the topic invites a close investigation.

Such an investigation meets with a number of immediate problems, some of them leading to difficulties that make them elusive targets in the short term. In order to make progress, it is first of all necessary to frame the problem in ways that allow analysis yet capture the essence of the problem to a degree that does not render the conclusions trivial or useless.

Two of the major issues are the following:

1. The sample space of spectral reflectance factors has never been defined so as to establish a general concensus in the literature. The topic is rarely approached other than in an ad hoc manner (for instance, by Wyszecki and Stiles [4]). The recent literature tends to use linear methods (like principal component analysis) on the set of spectral reflectance factors, a method that is not even consistent because of the fact that linear methods allow nonphysical reflectance factors (outside of the proper domain $[0,1])$.

2. The sample space of colors is taken very differently by various authors. Thus the mapping from spectra to colors is often ill defined, despite being of crucial importance. Here one encounters a variety of conceptual issues. One is that mappings on "psychologically uniform" spaces [9] are a priori suspect because they might well introduce a vicious circle. Moreover, such spaces involve non-linear mappings, rendering the analysis highly opaque. In discussions of the prior distributions of colors, the mapping from spectra on colors is crucial. There is only one choice that renders this map simple, and that is to use a colorimetric color space like the CIE $x y z$, for this renders the mapping linear. In this paper "color space" will invariably mean a colorimetric color space, though the CIE $x y z$ is awkward for a number of reasons (see below).

In this paper the aim is to pose the problem in the simplest and most transparent manner, drawing only on basic physics and purely affine colorimetry (conceptually not going beyond Maxwell's methods [10] and being fully objective; see below), thus enabling general conclusions to be drawn from the analysis. It is shown that very nonuniform hue statistics result from spectral reflectance statistics that are stationary with respect to shifts along the wavelength axis. This accounts for the apparent nonuniformity found in extensive statistical studies of databases of natural reflectance spectra [8].

\section{THEORY OF STATISTICS OF SPECTRA}

In the context of daily life transactions with the environment [11] one predominantly deals with "object colors" 
[12]. Main exceptions are the colors of light sources (e.g., the sky) and/or specularities. A useful simplification is to consider the set of spectra scattered to the eye from Lambertian surfaces [13,14], normally illuminated by a single illuminant. Such spectra are determined by the illuminant (for the purposes of the paper the "illuminant" is characterized by the beam remitted by a Lambertian surface of unit albedo) and the spectral reflectance factors [15]. Various generalizations such as oblique illumination and/or viewing, non-Lambertian BRDF's [16], and illuminant variations over a scene are immediate, though occasionally complicated. Even though evidently a simplification, this assumption suffices for the present purpose.

Notice that relaxation of this constraint would have a number of consequences that would require a redefinition of the problem: colors would depend upon both the direction of illumination (even for the same spectral illuminance) and the viewing direction, and thus it would no longer be possible to construct a "space of object colors" in a useful sense. This constraint is a necessary one in many investigations, even if not (what is common enough) explicitly mentioned. It is because the present aim is a principled, quantitative analysis that the constraint is explicitly introduced here, even though it can be relaxed significantly in practice.

Illuminant spectra are in principle constrained only through non-negativity of the spectral radiant power. In practice, naturally occurring illuminant spectra are fairly smooth and often close to Planckean spectra [17]. In this paper the illuminant will simply be fixed to a Planckean spectrum [17] of $5700{ }^{\circ} \mathrm{K}$, that is, roughly "average daylight." This is a very broad, almost flat spectrum; thus the illuminant will not introduce pronounced variations of hue. The precise choice is largely irrelevant, e.g., the assumption of a truly flat spectrum would not affect the conclusions at all nor would the adoption of an actual daylight spectrum. A daylight-like spectrum is preferable because of the likely evolution of the visual system.

Spectral reflectance factors of Lambertian surfaces $[13,14]$ are constrained to the range of zero to one. There is no obvious known constraint on the spectral variation; for instance, rare-earth glass powders have very highly articulated reflectance spectra [18]. However, even a cursory glance at databases of natural spectral reflectances reveals that generic articulations are structurally highly limited in their complexity.

\section{A. Homomorphic Mappings}

The fact that illuminant spectra are non-negative and reflectance spectra are limited to the range of zero to one implies that the simplest statistical models, e.g., Gaussian random processes, are not applicable. Worse still, no linear statistical methods are applicable. This is a major problem with conventional studies available from the literature, which are commonly based upon principal components analyses [19-21]. Such linear methods strictly make no sense because linear combinations of principal components do not respect the non-linear constraints that characterize the spectra.

When a model of the relevant physics is available, one should preferably conduct the analysis in the physical domain, where linear methods generally make sense. An example is the Kubelka-Munk theory [22,23] of turbid layers, where the physical parameters are the specific scattering and absorption coefficients and the layer thickness. These parameters are non-negative, whereas their values depend upon the choice of physical units. Thus the statistical model should be based on the distributions of logarithms of the values (Jeffrey's Law $[24,25]$ ). The physics lets one move from the parameter values to the reflectance factors. It is indeed standard practice in reflection spectroscopy [18] to study the spectral signature $\mathrm{k} / \mathrm{s}$ $=\left(1-r^{2}\right) / 2 r$ rather than the-immediately observedreflectance $r$, because this non-linear function of $r$ represents the absorption coefficient $k$ divided by the scattering coefficient $s$.

In general, however, no unique model of the physics is implied; thus one is forced to apply phenomenological methods. A simple approach to the problem is to distinguish between a nominal (supposedly linear) physical domain and the phenomenological domain of reflectance factors. The relation between these domains is some nonlinear transformation picked for convenience, i.e., of simple analytical form and invertible. (Any reasonable choice will be preferable over nothing.) For the sake of illustration consider the single-parameter case. Let the physical parameter be denoted $x \in \mathbb{R}$, the reflectance factor $r \in \mathbb{I}^{1}$ (where $\mathbb{I}^{1}=[0,1]$ ) and $r=F(x)$. Then an example of a convenient transformation is

$$
F(x)=\frac{1}{2}(1+\tanh x)
$$

with

$$
F^{(-1)}(r)=\operatorname{arctanh}(2 r-1) .
$$

Now one may use the "homomorphic mapping" method $[26,27]$ so as to define linear combinations of reflectances $(\alpha, \beta \in \mathbb{R})$ :

$$
r=\alpha r_{1} \oplus \beta r_{2}=F\left(\alpha F^{(-1)}\left(r_{1}\right)+\beta F^{(-1)}\left(r_{2}\right)\right),
$$

and so forth. It is guaranteed that $r \in \mathbb{I}^{1}$ if $r_{1,2} \in \mathbb{I}^{1}$. The $\oplus$ operator in the phenomenological domain corresponds to simple addition (+) in the physical domain. Using the homomorphic mapping linear methods such as principal components analysis becomes a valid option, for the basis of principal components may be set up in the physical domain.

In this setting simple statistical models of reflectances become viable, e.g., the physical parameter can be taken from some Gaussian random process. Notice that even in this simple case a histogram of reflectance factors might well turn out to be bimodal. Although the statistics looks simple in the physical domain, it looks complicated in the reflectance domain. When faced with databases of natural reflectances, it is this complexity that strikes one initially. The preferred way to handle this complexity is to search for a suitable physical domain in which the statistics is simple. 


\section{EMPIRICAL STATISTICS OF SPECTRA}

\section{A. Generalities}

The available data on natural reflectance spectra are sparse. There are a limited variety of databases in the public domain. These databases are of very different natures. Here only a few of the better known and easily available instances are considered. The earliest is probably the well-known Krinov [28] database, which will not be considered here. A useful overview of modern, publicly available databases is given by Kohonen et al. [29]. The databases considered here are more fully described in their paper.

The Dupont painted chips, the colored papers, and the Munsell chips are designed for (often vivid) color and are likely to be colored by means of a rather limited range of pigments. Such databases are rather unlikely to be ecologically valid. The "Finnish flowers" database probably offers a representative sample, though of a limited domain (vegetation and flowers overrepresented). The "NCSU objects" are selected from the daily environment without obvious rationale. This makes this set of much interest, despite the fact that it (necessarily) undersamples the daily environment grossly. Thus the Finnish flowers and NCSU objects databases may be said to have at least some ecological validity, although biased and sparse. These data are far from ideal. In order to attempt a statistics of object colors, one needs far more extensive and homogeneous data. In practice the only viable way to proceed is to model the statistics of the available sets, so as to be able to generate novel instances ad libitum.

Since the visual range is very narrow (relative bandwidth [30] about 0.24) it seems reasonable on physical grounds to assume $a$ priori that the distribution of object reflectance spectra is stationary, i.e., does not significantly depend upon wavelength. Then the databases can be used to judge such properties as the distribution of reflectance values and the nature of spectral articulation, whereas the generator of instances enforces stationarity.

\section{B. Databases}

The histograms of reflectance factors as sampled from available databases in the nominal physical domain are fairly close to normal for reasonable homomorphic transformations (see Fig. 1). Some problems derive from reflec-

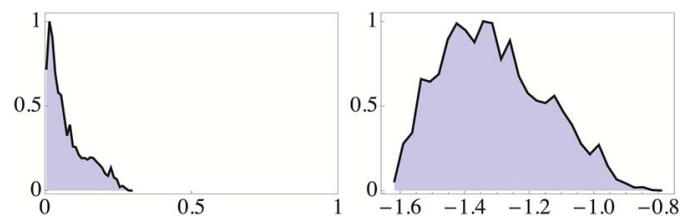

Fig. 1. (Color online) Left: histogram of reflectance factors (all samples, all wavelengths pooled) from the NCSU objects database. The histograms are normalized to a maximum value of unity. This is the histogram in the "phenomenological domain" (range of the reflection factor between zero and one). Notice that the histogram is almost exponential. Right: the same data in the "physical domain" (range minus to plus infinity). Though the histogram is still a little skew, it is much more centrally symmetric than the one at left, as well as defined on the full axis. A simple normal distribution captures much of the structure of the available data bases. In all cases the flanks of the distribution are ill defined due to observational errors (actual reflectance factors too close to either zero or one).

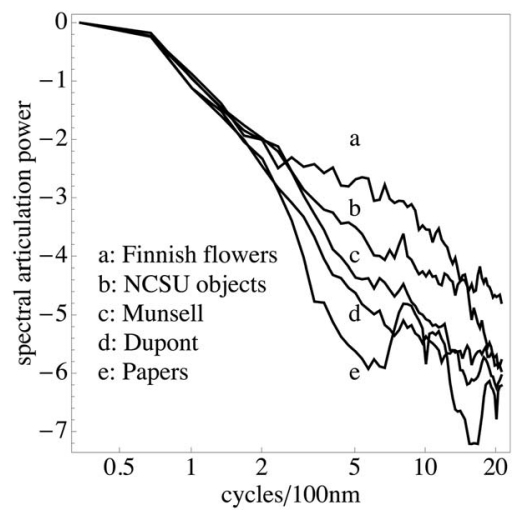

Fig. 2. Fourier spectral power of the spectral envelope of the radiant power falls by (about) the inverse second power of the frequency. The frequency is given in terms of the number of cycles per $100 \mathrm{~nm}$ on a logarithmic scale. Notice that the "visual range" measures about $200-400 \mathrm{~nm}$. This is a double logarithmic plot: on the abscissa the actual values are indicated; on the ordinate the values of log spectral power (base 10). (Notice that 1 decade on the abscissa has the length of 4 decades on the ordinate; thus the slope is about -4$)$.

tance values near zero or unity that are rather suspect (because of technical problems) for virtually all of the available databases. Normal distributions fit the available databases sufficiently well.

The spectral articulation of natural reflectance factors has rarely been studied. In Fig. 2 the spectral articulation power (not radiant power!) spectrum of the reflectance factors in the physical domain is shown in a log-log plot. The usual precautions are observed, i.e., the linear trend is subtracted and suitable windowing applied. The conclusion is that typical reflectance spectra are not very articulate and the number of transitions is small and falls steeply with their number. This will turn out to be a key issue.

A generator of random spectral reflectance factors that yields samples not significantly distinct from the items in any given database need not be complicated; a rather limited number of parameters suffices. The simplest algorithm will have at least these three parameters: level and width of the amplitude distribution in the physical domain and slope of the log-log power spectrum. Various additional parameters are feasible but are superfluous for the purpose of this paper as they would not affect the nature of the conclusions. Examples presented below are due to a Gaussian random signal with level -1.0, amplitude (standard deviation) 1.2 [both in terms of Eqs. (1) and (2)], and spectral slope -1.8 (in the power spectrum double that) in the physical domain. This is a reasonable fit to all of the databases. In Fig. 3, six arbitrary samples are shown. The precise values quoted here are of no relevance to the final conclusions; they merely serve to enable a numerical illustration of the predictions from formal analysis.

It is important to appreciate the fact that the only properties that matter to the final conclusions of this paper are that

- the statistics of reflectance spectra is invariant with respect to translations along the wavelength axis, owing 

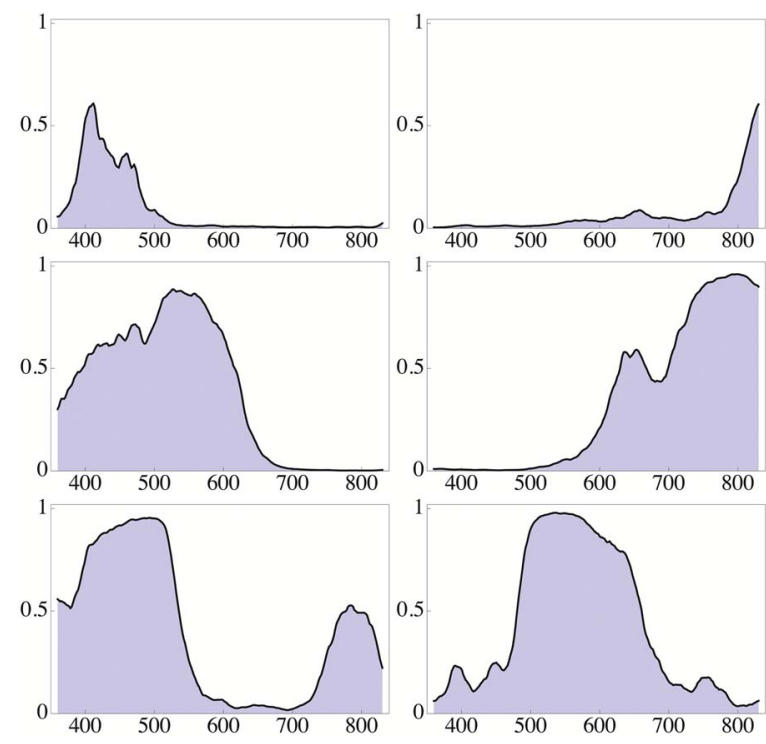

Fig. 3. (Color online) Six samples from randomly generated spectral reflectances. The parameters of the random generator have been selected so as to simulate the statistics of typical databases of "natural reflectance spectra." This random generator has stationary statistics; there is no wavelength preference whatsoever.

to the narrow width of the visual region (thus there are no significant wavelength preferences in the natural environment);

- reflectance spectra are generically smooth in the sense that the power spectrum of the reflectance spectral envelope falls off as a power (of roughly 2) of the frequency.

The second property essentially guarantees the occurrence of vivid colors (see below) and is thus a prerequisite for the very utility of human-like color vision. More specific properties of the statistics are needed only to enable numerical simulations as illustration of the formal conclusions.

\section{PROJECTION OF REFLECTANCE SPECTRA ON COLOR SPACE}

\section{A. Generalities}

Different from the physics of reflectance spectra, which involves essentially non-linear phenomena (such as the Kubelka-Munk theory), colorimetry has a natural linear structure. Colors are equivalence classes of mutually indiscriminable radiant power spectra, the equivalence being induced by a linear projection on a mere three dimensions (trichromacy).

In this paper I will use the conventional CIE $196410^{\circ}$ observer [31,32] and a Planckean spectrum of $5700^{\circ}$ illuminant. This settles the gamut of possible object colors: it is the Schrödinger color solid [33,34]. An "object color" is defined as [34]

$$
\mathbf{c}\left(r_{\lambda}\right)=\int_{0}^{\infty} r_{\lambda} I_{\lambda} A_{\lambda}^{T} \mathrm{~d} \lambda,
$$

where $r_{\lambda}$ denotes the spectral reflectance factor, $I_{\lambda}$ the radiant spectral power of the illuminant, and $A_{\lambda}$ the color matching matrix (an $\infty \times 3$ matrix). Since all object spectra $r_{\lambda} I_{\lambda}$ are dominated by the spectrum of the illuminant, they fill a hyperparallelepiped in the space of spectra [35], and since the color solid is a linear projection [36] of this hyperparallelepiped in color space, it is a centrally symmetric convex body. Infinitesimally near the black point the color solid is simply the spectrum cone, and by symmetry it must look like the inverted spectrum [37] cone infinitesimally near the white point.

At the boundary of the color solid the reflectance spectra are "optimal colors" [34]; that is the spectral reflectance is either zero or one with no more than two transitions throughout the spectrum. This means that any color on the boundary can be obtained as the difference of two (Goethe) "edge colors," which are optimal colors with only a single transition [34,38]. The edge colors come in two varieties, short-wavelength-pass and long-wavelengthpass. Because of the central symmetry, one needs only the short-wavelength-pass edge color series to construct the complete color solid. This series is

$$
\mathbf{s}(\lambda)=\int_{0}^{\lambda} I_{\lambda} A_{\lambda}^{T} \mathrm{~d} \lambda ;
$$

its locus in color space is a generic spiral (non-negative curvature, non-negative torsion) of one turn connecting the black to the white point. The corresponding color sequence is $\mathrm{K}$ (black), $\mathrm{B}$ (blue), $\mathrm{C}$ (cyan) and $\mathrm{W}$ (white). (The formal meaning of the use of hue names in the context of pure colorimetry is explained below; at this point they are used merely for descriptive convenience.) The complementary series (long-wavelength-pass edge colors) is $\mathrm{W}, \mathrm{Y}$ (yellow), R (red), and K. Notice that G (green) and M (magenta) are not among the edge colors, since they imply two spectral transitions. Notice that $\mathrm{G}$ is a "spectral color" [39] (band pass), whereas M (band stop) is "non-spectral" [37].

\section{B. Canonical Projection}

On informal observation the Schrödinger color solid [34] appears as a slightly rounded, or "inflated," and skewed cube [40]. Only affine differential geometry [41,42] applies to color space, whereas the notion of "cube" is metrical. However, when a linear transformation (change of primaries) turns the color solid "visually" into an "almost" cube (Fig. 4), the relations in that representation might be defined as metrical. Below, this approach is given a formal basis; it is intrinsically different from the approach of Cohen in Cohen and Kappauf [43], who implicitly introduces an ad hoc metric on the space of spectra [44].

The principled approach is to construct the inscribed parallelepiped of maximum volume. A parallelepiped with one vertex at the origin, for ease of reference designated "crate," is defined through a trisection of the spectrum $[45,46]$ into S (short-wavelength), M (mediumwavelength), and $\mathrm{L}$ (long-wavelength) ranges. (This scheme was introduced by Schopenhauer [45] who spoke of L, M, S "parts of daylight.") Notice that the corresponding colors are optimal colors that lie on the boundary of the color solid, as do their sums and differences. Together these define the vertices of a "crate" (linearly deformed cube with one vertex at the origin) inscribed into the color 


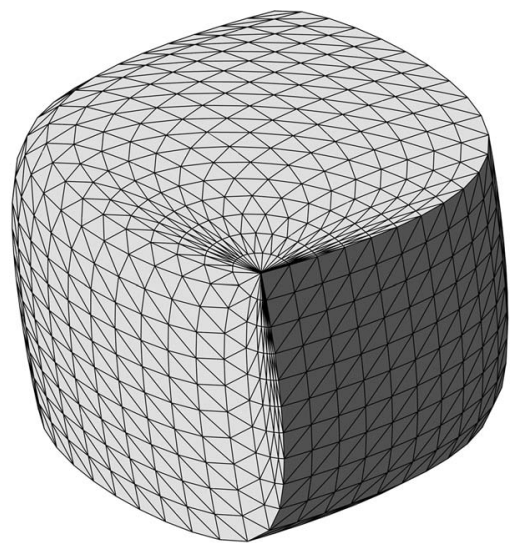

Fig. 4. The Schrödinger color solid looks like a "slightly inflated cube" when represented in the canonical coordinates introduced later in the text. (In the CIE $x y z$ system it looks rather less symmetrical.) It is composed of two smooth patches (the spectral and the non-spectral optimal colors) that are mutually congruent by central symmetry and hang together via two sharp creases (the edge color loci) and two conical points (the white and black points).

solid. The ratio of the volume of this crate to that of the color solid is invariant against linear transformations [47]; thus it makes good sense to search for the crate of maximum volume as the best approximating inscribed crate of the color solid. It can be found by exhaustive search for the two spectral-cut loci and turns out to be unique. (The cut loci are 483 and $567 \mathrm{~nm}$, but of course they depend upon the illuminant spectrum.) Given the maximum volume crate [48], one constructs the unique linear transformation that turns it into the unit cube. The dominant wavelengths of the short, medium, and long spectral parts (respectively 445, 527, and $601 \mathrm{~nm}$ ) prove to be close to those of unique [49] blue, green, and red, or the "prime colors" of Thornton [50]; thus one may speak of the "RGB cube" [51]. Here R (red), G (green) and B (blue) are the colors of the spectral ranges $\mathrm{L}, \mathrm{M}$, and $\mathrm{S}$, respectively. (The hue names are very descriptive, which is why they are used, but they stand only for colors (in the colorimetric sense!) of spectral regions and not for qualia. Whoever has objections to this can easily translate all hue names occurring in this paper into references to colors of well-defined spectral regions.) Other vertices of the cube are $\mathrm{K}$ (the origin) and $\mathrm{W}$ (white, the color of $L \cup M \cup S$ ) and $\mathrm{C}$ (cyan, color of $M \cup S$ ), M (magenta, color of $L \cup S$ )
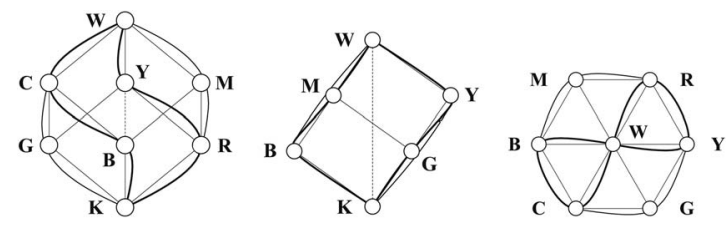

Fig. 5. RGB cube, Schrödinger color solid, and edge color loci in the canonical projection, shown as viewed from three mutually orthogonal viewing directions (at far right the gray axis, at far left a direction in the yellow-blue plane, at center a direction in the red-cyan plane). This structure is the very backbone of object color space. Notice that the edge color loci closely follow the KRYW and WCBK edge progressions of the cube, whereas the closed YGCBMR edge progression approximates the "color circle," that is, the locus of colors maximally removed from the gray (KW) axis.

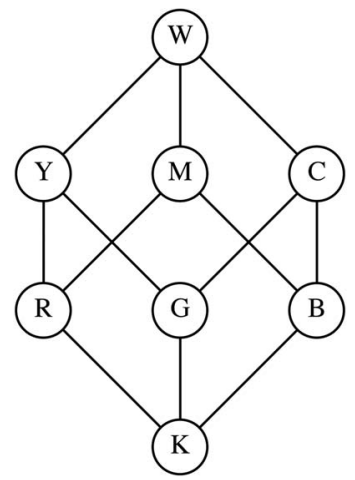

Fig. 6. Hasse diagram of the partial order of cardinal colors by set inclusion. Notice how it looks like a skeleton projection ("wireframe rendering") of the RGB cube.

and $\mathrm{Y}$ (yellow, color of $L \cup M$ ). The closed edge progression YGCBMR is a non-planar hexagon that has the topology of the color circle. (see Fig. 5.)

Notice that the six cardinal colors, consisting of the three primary colors Red, Green, and Blue and the three secondary colors Cyan, Magenta, and Yellow, are not only colors, but also (reflectance) spectra. Moreover, they are special spectra because they are "parts of daylight," that is, equal to the illuminant spectrum over certain ranges of the wavelength domain and zero elsewhere. These spectral ranges are naturally ordered by set inclusion. For instance, white contains yellow (for white is yellow plus blue) and yellow contains red (for yellow is red plus green). The Hasse diagram [52] of the partial order $[53,54]$ is shown in Fig. 6. Notice that it looks like a skeleton representation of the RGB cube. This is not a coincidence at all; the partial order of inclusion implies the geometrical structure of the RGB crate. Inverting this, the familiar notion of the RGB cube as an approximation to the object color solid (of immense importance to computer graphics [55]) implies the Schopenhauer trisection of the visual range into parts of daylight.

In Fig. 7 the chromaticity diagram is plotted as an equilateral RGB triangle with the white point at its bary-

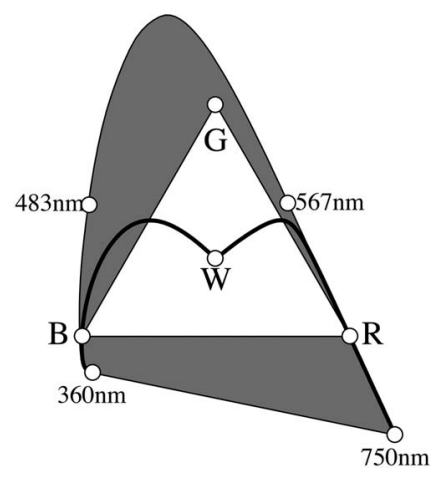

Fig. 7. Equilateral RGB triangle is the preferred (most symmetrical) chromaticity diagram in the RGB representation. Notice that the spectrum locus runs far outside the triangle. This might easily lead to erroneous impressions, however; the volume of the Schrödinger color solid that is mapped outside the triangle is only about $8 \%$ of the total volume. It is very rare (probability less than $1 \%$ ) for a color from one of the databases to fall outside the RGB triangle. The wavelengths $483 \mathrm{~nm}$ and $567 \mathrm{~nm}$ are the cut loci. Notice that the spectrum locus represents the chromaticity of the black point. The fat curves are the edge color loci. 
center [56]. This is the preferred representation since it treats the primary colors equally. The region in the exterior of the triangle may safely be ignored, since essentially all natural colors will map into the interior. The exterior represents (despite appearances!) only $8 \%$ of the total volume of the Schrödinger color solid [57]. Moreover, the colors mapping to the exterior are so close to optimal colors as to be highly unlikely to be encountered in reality.

The RGB coordinates of object colors are very nearly in the $[0,1]$ range. Minor under- and overshoots occur for colors near the color solid boundary. Such colors are very rare in nature, and in practice almost all colors defined by the databases fall well within the interior of the cube [58]. In practice one may use this to translate spectra into RGB colors of display units (occasionally doing minor clipping to keep values in range) with excellent results. Indeed, typically (that is, in the absence of under- or overshoot) such display colors perfectly replicate the photon catches in the retinal cones and thus the input to the brain. Thus the representation is a perceptually natural one (much more so than CIE $x y z$ coordinates), whereas-from the perspective of physics-it represents "colors" as observations in coarse-grained spectroscopy (using only three spectral bins, the $\mathrm{S}, \mathrm{M}$, and $\mathrm{L}$ regions). This renders the representation ideally suited for the intended statistical analysis.

The RGB colors can be parsed easily in the manner of Ostwald [59]. For example, consider the color $\mathbf{x}$ $=\{0.8,0.6,0.3\}$. The first thing to do is to write $\mathbf{x}=0.3 \mathbf{w}$ $+0.2 \mathbf{k}+0.5 \mathbf{f}$, where $\mathbf{f}=\{1,0.6,0\}$. The color $\mathbf{x}$ is written as an amount (30\%) of white, an amount (20\%) of black, and an amount (50\%) of color, in this case the color f. Notice that a color such as $\mathbf{f}$ necessarily lies on the YGCBMR color circle and is always an interpolation between a primary color ( $\mathrm{R}, \mathrm{G}$, or $\mathrm{B}$ ) and a (neighboring) secondary color $(\mathrm{C}, \mathrm{M}$, or $\mathrm{Y})$. In this case one has $\mathbf{f}=0.4 \mathbf{r}+0.6 \mathbf{y}$, that is, $40 \%$ red and $60 \%$ yellow, a yellowish orange. This description yields an immediate handle on the perceived color ("yellowish orange with about a third white and a fifth black") and simultaneously a handle on the spectral reflectance factor (30\% uniform spectrum, plus $30 \%$ yellow addition plus $20 \%$ red addition); see Fig. 8 . Of course, this spectrum is only an approximation since you may add arbitrary metameric blacks to it. However, it is usually a good approximation when the color is near the color solid boundary (because there is no room for additional articulation) and because most metameric blacks are highly unlikely, anyway, owing to their (necessarily) high spectral articulation.

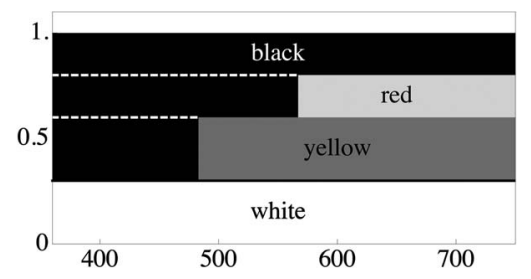

Fig. 8. Spectrum of the color $\mathbf{x}=\{0.8,0.6,0.3\}$ can be conceived of as addition of a uniform spectrum (white), nothing (black), yellow (extending over the red and green regions), and red (extending over the red region). Thus any color is the convex combination of white, black, and a "full color," that is, the interpolation between a primary color and one of its nearest secondary colors.
Since the RGB cube is uniquely determined (at least if the illuminant spectrum is fixed), one may use it as a canonical representation of object colors. The $\mathbf{r}, \mathbf{g}$, and $\mathbf{b}$ vertices are the colors of the $\mathrm{S}, \mathrm{M}$, and $\mathrm{L}$ spectral regions and may be taken as the primaries. The color circle YGCBMR is a well-tempered scale in the sense that each vertex has the same dominant wavelength as the equal mixture of its two neighbors; for instance, $\mathbf{y}$ has the same dominant wavelength as $\frac{1}{2}(\mathbf{r}+\mathbf{g})$, and so forth. Thus the scale is uniform in the sense of Ostwald's "Principle of Internal Symmetry" [59-61] (which can be used to further subdivide the scale), used in the construction of the Ostwald atlas [62] and the German DIN system [63]. It is close to various eye measure [64-66] systems [67] such as the Munsell system [68]. In fact, analysis shows that the scale falls well within the mutual variations of the various eye measure systems. This is convenient, as it implies that one need not use any (by nature arbitrary) eye measure system. This avoids the possibility of vicious circularity mentioned in the introduction.

One may set up a metric for color space as the standard Euclidean metric in the unit cube. This is useful because it allows one to put a colorimetrically relevant scale on the wavelength axis, namely, the arclength of the edge color sequence. Since the boundary of the color solid can be constructed from the edge color locus (it is a surface of translation [69] in two ways), such a metric is a very natural one. Since the finite length of the edge color locus parameterizes the full wavelength domain (of infinite length!), the parameterization is obviously very uneven. This unevenness is bound to show up in color statistics, because the physics of spectral reflectances is translationally invariant along the wavelength axis, whereas the differences of the corresponding colors are more immediately related to distances in the RGB cube.

\section{Statistics of Object Colors}

Perhaps the simplest, intuitive model is the following: Colors are observations in coarse-grained spectroscopy using three adjacent spectral bins. For simplicity assume these bins to be of equal size. Reflectance factors are random telegraph waves, toggling between zero and one, with a rather limited number of transitions in the visual range. For simplicity assume $n=0,1,2 \ldots$ transitions with steeply decreasing probability as the number goes up. Assume a uniform distribution for the transition wavelengths, thus no spectral selectivity in the physics. Notice that this models both the visual system (the three bins) and the generic spectral reflectances (the random telegraph waves), both in a very coarse manner; the model is almost a caricature.

However, the particular model of the visual system is quite apt since it captures the RGB structure derived above. In particular, for every spectrum there exists a metameric spectrum that is exactly constant over the three bins. Likewise, the random telegraph waves capture much of the essence of actual reflectance spectra. That this very simple, schematic model captures the essence of the problem is evident from the fact that more elaborate models yield qualitatively similar results (see below). The advantage of the model is that it allows one to grasp the essentials in an intuitive manner. 
Granted the model, consider the corresponding distribution of colors: In the case of zero transitions, one obtains only W (white) and K (black); in the RGB cube only two vertices are hit. Thus the distribution is not over the volume of the RGB cube but is discrete, over lowerdimensional subsets. This is the general pattern. One obtains uniform distributions over convex hulls of subsets of vertices. For instance, for $n=1$ only certain edges are populated and for $n=2$ only certain faces, whereas for $n$ $=3$ or more, parts of the volume are hit. Because there is a finite probability to hit certain vertices or edges, the probability density becomes infinite at these loci.

Because six of the edges (those containing $\mathrm{W}$ or $\mathrm{K}$ ) have uniquely defined dominant wavelengths, the probability density in the dominant wavelength domain "explodes" at these wavelengths. Thus one obtains a very non-uniform distribution of colors and dominant wavelengths, even though the statistics of the spectra is stationary (Fig. 9).

The deciding factor is the dimensionality of the subsets. Planar distributions are infinitely denser than volume densities, linear densities infinitely denser than planar densities, and point densities infinitely denser than linear densities.

Notice that vivid colors imply a small number of transitions. For a random telegraph wave with a very large number of transitions $n \gg 1$ the resulting color is median gray $\left(\left\{\frac{1}{2}, \frac{1}{2}, \frac{1}{2}\right\}\right)$ with only a little noise of the order $1 / \sqrt{n}$ $\ll 1$ on the coordinates. Thus high-frequency wriggles of the spectral reflectance (or illuminant spectrum) are utterly irrelevant to the issue.

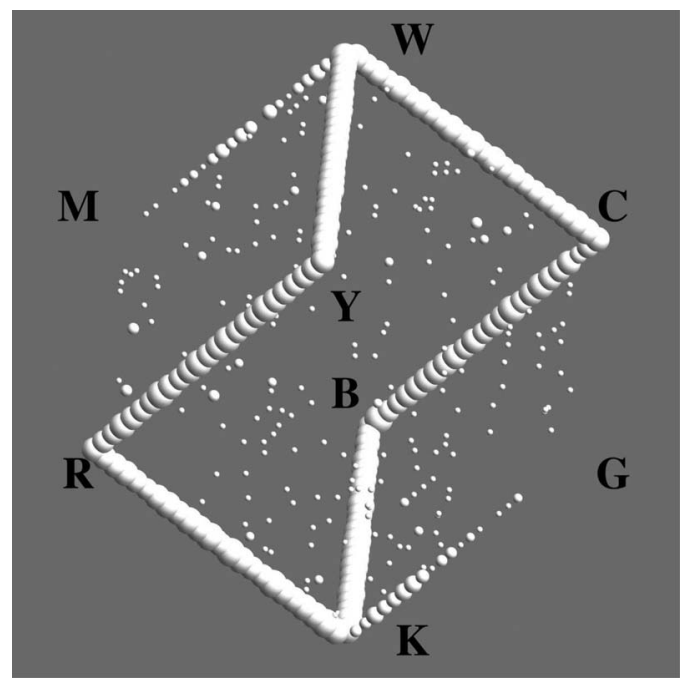

Fig. 9. Result of a simulation of the simple model. The number of transitions was $0,1,2, \ldots$ with probability $\frac{1}{2}, \frac{1}{4}, 1 / 8 \ldots$ Of 10,000 samples about 5000 ended up at either the white or the black point; these are not represented here. The spheres represent samples of volume density over volumes of about one thousandth of the volume of the RGB cube; the radius is taken proportional to the logarithm of the density. Notice that almost all samples end up on either the long-wavelength-pass edge color locus (KRYW edge sequence) or the short-wavelength edge color locus (WCBK edge sequence). Some density accumulates on the MW edge (near W) and on the GK edge (near K). The remaining density concentrates on the surface of the RGB cube with only a sprinkle of samples in the interior. Thus the most frequent object colors are R and B (dark), C, and Y (light), with some representation of dark $\mathrm{G}$ and light $\mathrm{M}$. Thus the simple model produces very pronounced non-uniformities.
For reasonable parameters, one obtains the highest density on the white and black points, next-higher density on the edge color loci (KRYW and WCBK edge progressions), next-higher density on the faces, and lowest density in the interior of the RGB cube. Thus the most common colors are white and black, followed by red, blue, yellow, and cyan, followed by oranges (red-yellow mixtures) and turquoises (blue-cyan mixtures), followed by darkish greens and light purples, followed by ..., etc. These densities will be modulated by the nature of the mapping of wavelengths on edge color arclength, effectively lowering the densities for the blues, cyans, and purples. This already explains the bulk of the nonuniformities found empirically (see below).

Since the RGB cube is such an excellent model for the Schrödinger color solid and all colorful colors are due to spectra close to optimal colors, realistic simulations (involving a statistical model of reflection spectra based on the databases and the CIE 1964 color matching functions) reveal a very similar pattern (see Section 5). This pattern is very similar to that found (with considerable effort; e.g., see [8]) via the available databases. Apparently the simple factors identified here already suffice to account for these findings. Even the simple model does all that is required for understanding. The major addition of the exact treatment is due to the non-trivial relation of the wavelength scale to the arclength of the edge color locus, a minor additional factor.

\section{Distribution of Optimal Colors}

The boundary of the color solid can be parameterized by the transition wavelengths of the optimal colors, $\left\{\lambda_{1}, \lambda_{2}\right\}$ (say). The convention that $\lambda_{1}<\lambda_{2}$ denotes spectral colors and $\lambda_{1}>\lambda_{2}$ the non-spectral colors is convenient. Then $\lambda_{1}=0$ specifies the short-wave-pass edge colors and $\lambda_{2}=\infty$ the long-wave-pass edge colors. A uniform generator of random optimal colors simply generates pairs of random wavelengths. Such colors will be mainly blacks and whites except when at least one of the wavelengths happens to fall within the visual region. Since all vivid colors are almost optimal colors, it is of interest to consider the probability distribution over the boundary of the color solid.

The optimal colors $\left\{\lambda_{1}, \lambda_{2}\right\},\left\{\lambda_{1}+\mathrm{d} \lambda_{1}, \lambda_{2}\right\},\left\{\lambda_{1}, \lambda_{2}+\mathrm{d} \lambda_{2}\right\}$, and $\left\{\lambda_{1}+d \lambda_{1}, \lambda_{2}+d \lambda_{2}\right\}$ subtend a paralellogram of area $J\left(\lambda_{1}, \lambda_{2}\right) \mathrm{d} \lambda_{1} \mathrm{~d} \lambda_{2}$ (say). The function $J\left(\lambda_{1}, \lambda_{2}\right)$ is of immediate interest because its reciprocal is proportional to the density of optimal colors for the uniform random generator. This function can immediately be obtained (numerically) from the CIE color matching functions and the illuminant radiant power spectrum.

A spectral optimal color is given by

$$
\mathbf{i}\left(\lambda_{1}, \lambda_{2}\right)=\int_{\lambda_{1}}^{\lambda_{2}} I_{\lambda} A_{\lambda}^{T} \mathrm{~d} \lambda=\mathbf{s}\left(\lambda_{2}\right)-\mathbf{s}\left(\lambda_{1}\right),
$$

in terms of the short-wavelength-pass edge colors [Eq. (5)]. Thus one has

$$
\frac{\partial \mathbf{i}\left(\lambda_{1}, \lambda_{2}\right)}{\partial \lambda_{1}}=-\left.\frac{\mathrm{d} \mathbf{s}}{\mathrm{d} \lambda}\right|_{\lambda_{1}}=-\mathbf{m}_{\lambda_{1}},
$$




$$
\frac{\partial \mathbf{i}\left(\lambda_{1}, \lambda_{2}\right)}{\partial \lambda_{2}}=+\left.\frac{\mathrm{d} \mathbf{s}}{\mathrm{d} \lambda}\right|_{\lambda_{2}}=+\mathbf{m}_{\lambda_{2}},
$$

where $\mathbf{m}_{\lambda}=I_{\lambda} A_{\lambda}^{T}$ denotes the color of a "monochromatic beam" (Newton's [39] "homogeneous light") of wavelength $\lambda$, a generator of the spectrum cone. One notes in passing that the tangent plane at the color solid is parallel to the plane spanned by the spectrum cone generators $\mathbf{m}_{\lambda_{1}}$ and $\mathbf{m}_{\lambda_{2}}$. Thus the spectrum cone is indeed tangent to the color solid as mentioned earlier. Moreover, the most vivid optimal colors must be farthest from the achromatic axis; thus the tangent plane must be parallel to the achromatic axis, implying that $\lambda_{1}$ and $\lambda_{2}$ are mutually complementary. These most vivid colors are Ostwald's "full colors" (German: Vollfarben; they populate the color circle of the Ostwald color atlas [60].)

Thus the First Fundamental Form [47] (metric) of the surface of the boundary solid is (in the conventional notation of the differential geometry of surfaces)

$$
\mathrm{d} s^{2}=E \mathrm{~d} \lambda_{1}^{2}+2 F \mathrm{~d} \lambda_{1} \mathrm{~d} \lambda_{2}+G \mathrm{~d} \lambda_{2}^{2},
$$

with

$$
\begin{aligned}
& E=\mathbf{m}_{\lambda_{1}} \cdot \mathbf{m}_{\lambda_{1}}, \\
& F=-\mathbf{m}_{\lambda_{1}} \cdot \mathbf{m}_{\lambda_{2}}, \\
& G=\mathbf{m}_{\lambda_{2}} \cdot \mathbf{m}_{\lambda_{2}},
\end{aligned}
$$

and consequently the area element is

$$
J\left(\lambda_{1}, \lambda_{2}\right)=\sqrt{\operatorname{det}\left|\begin{array}{ll}
E & F \\
F & G
\end{array}\right|}=\left\|\mathbf{m}_{\lambda_{1}} \times \mathbf{m}_{\lambda_{2}}\right\| .
$$

Apparently $J\left(\lambda_{1}, \lambda_{2}\right)$ is given by the modulus of the cross product of the spectrum cone generators at the transition locations of the optimal color $\mathbf{i}\left(\lambda_{1}, \lambda_{2}\right)$, an intuitive and simple result. A numerical calculation (Fig. 10) re-

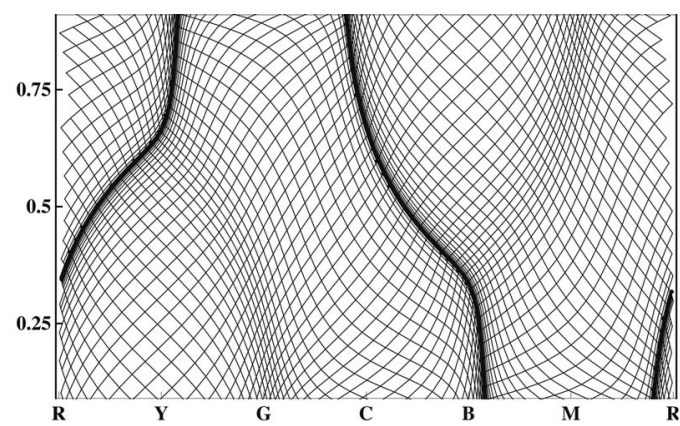

Fig. 10. Nexus of parameter lines for the passband center and passband width of the ideal colors ("slit location and width in spectroscopic terms"). The area of the meshes indicates the function $J$. Notice that $J$ becomes zero at the edge color curves (the area collapses to a curve). Here the boundary of the color solid has been plotted in cylinder coordinates: vertically the "height" in the achromatic dimension (bottom horizontal edge represents the black point, top horizontal edge the white point), and horizontally the azimuth, which has been marked with the cardinal colors of the color circle. This is the type of plot conventionally shown in discussions of color preferences. The plot implicates a preponderance of dark reds and blues, light yellows, and cyans as well as (though less pronounced) dark greens and light purples. veals a pattern that is in all respects qualitatively similar to that found for the simple RGB cube model. A uniform (in terms of wavelength) optimal-color random generator yields very non-uniformly distributed colors.

\section{DISTRIBUTION OF COLORS FOR STATIONARY STATISTICS EMULATING CERTAIN COLLECTIONS OF "NATURAL SPECTRAL REFLECTANCES"}

Although the results for the simple model (random telegraph waves and RGB cube approximation, Subsection 4.C) and the optimal colors (Subsection 4.D) should be compelling enough, it is perhaps of some interest to consider the distributions of colors for stationary statistics (translation invariant with respect to wavelength) that emulate the statistics of some of the available databases. The random reflectances are from the distribution illustrated in Fig. 3; a total of 10,000 samples were used for the study (far higher than the size of typical data bases, but of course there is really no limit when a random generator is used). In Fig. 11 a contour plot in the RGB cube illustrates the fact that most samples cluster on the edge color loci.

Plotted in the chromaticity diagram (Fig. 12), the clustering about the edge color loci is also obvious, as is the fact that only a small number of samples mapped on the exterior of the RGB cube.

A histogram of the hue angles (Fig. 13) reveals that dark colors cluster on red and blue, whereas light colors cluster on yellow and cyan. Notice that the red and blue peaks are far more evident than the yellow and cyan peaks. This depends on the parameters of the random generator. For instance, moving the level (in physical space) from -1.2 to zero serves to make the yellow and cyan peaks be just as evident as the red and blue peaks (indeed, then there is a complete symmetry between the dark and light colors). This illustrates the importance of the level. For all databases considered in this paper the level was negative (about -1.2, about the value used in the simulation, which is representative of these databases)

The influence of the amplitude is straightforward. As the amplitude is decreased, the colors move toward ach-

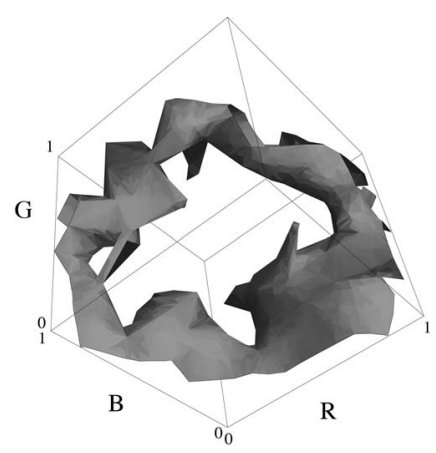

Fig. 11. Result of simulations 10,000 samples) with a random spectral reflectance generator with stationary statistics. The generator simulates generic databases of natural colors. In the contour plot one sees that the density is concentrated on the edge color loci. 


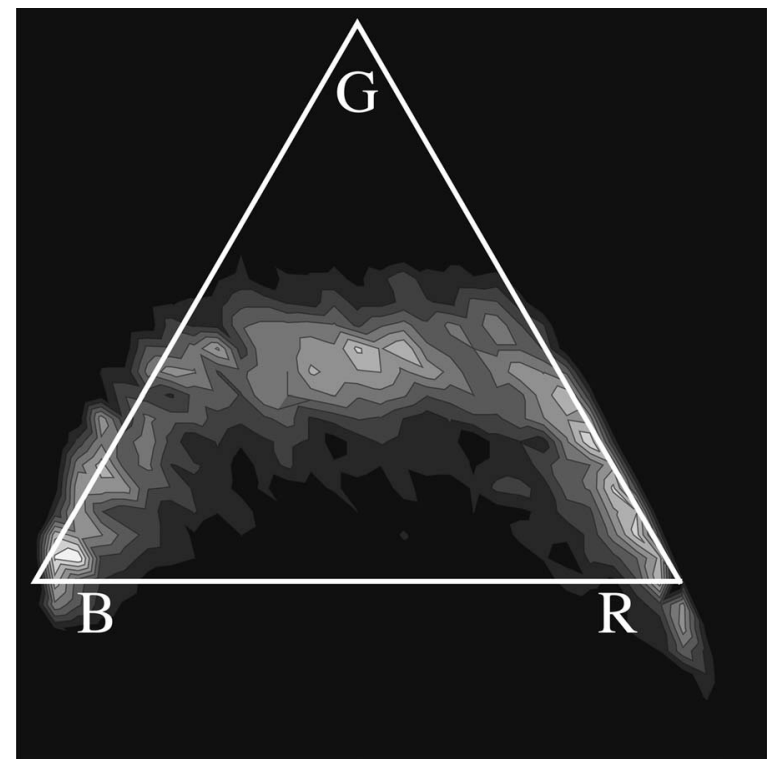

Fig. 12. Density of chromaticities for a random reflectance generator with stationary statistics. The parameters are such that the samples are similar to what one might obtain from a typical database of "natural reflectance spectra." The density is based on 10,000 samples. Notice that the density concentrates upon the edge color loci.

romatic; as it is increased, the colors move toward the boundary of the color solid (crowding on the edge color loci).

The influence of the spectral slope is crucial. The value used in the simulation is representative of all databases covered here. A steeper drop with increasing articulation frequency serves to boost the number of achromatic colors, whereas the vivid colors that remain cluster even more tightly on the edge color loci. A flatter spectrum increases the articulation, with the effect that colors strongly desaturate. As mentioned above, all random telegraph waves of sufficiently high articulation frequency yield the median gray color.

Thus the effective descriptive parameters of the databases are first of all the articulation spectral slope (something like the slope actually found is about optimal to arrive at vivid colors), then the amplitude (the value of about 1 is fine, higher would yield slightly more vivid colors), and finally the level. A level near zero yields the most extensive gamut, though different levels will still yield vivid colors if only the amplitude is large enough. There should be sufficiently many zero crossings in the physical domain. These parameters suffice to account for the facts.
Of course one might proceed to model any of the individual databases in exquisite detail, but this would really defeat the purpose. These simulations merely serve as illustrations of what one is likely to find with any extensive enough data base. The main conclusions are due to formal reasoning rather than to statistical simulations.

\section{CONCLUSIONS}

The detailed statistics of ecologically valid databases of object reflectance spectra turns out to be irrelevant to the issue of the prior statistics of object colors. Stationary statistics (no wavelength-selective properties) yields very non-uniform statistics of object colors with high densities near the edge color loci.

These conclusions do not depend upon the special representation of color space used in this paper nor on the details of the generator of random reflectances. The present representation was chosen because it serves to render many of the effects intuitive, if not obvious. A conventional representation is bound to yield the same results, but these are likely to appear surprising and perhaps due to details of the simulation. In fact the conclusions are highly robust against minor details and (especially) the simple model makes perfectly clear why.

The edge color loci are immediately due to the photopigment-action spectra. The latter determine the color matching functions, which are essentially linear combinations of the photopigment-action spectra, the particular combination being due to the (essentially arbitrary) set of primaries. The edge color loci are nothing but the cumulative color matching functions in the color space determined by the primaries, the only remaining freedom being the radiant power spectrum of the illuminant. In this paper the illuminant was fixed by assuming average daylight. The very non-uniform statistics of object colors with high densities near the edge color loci is ultimately due to the photopigment-action spectra. In studies like those of Philipona and O'Regan [8] there is no reason to assume that anything but stationary statistics is of relevance. To find signs of the ecological origins of object colors (which no doubt are present, too) would require an even more enormous effort than that of Philipona and O'Regan 2006 already was, because such modulations apparently drown in the very strong non-uniformities that are due to the structure of the visual system. This need not surprise one since the non-uniformities due to the visual system are singularities, whereas one expects ecological preferences to be gradually distributed. One would

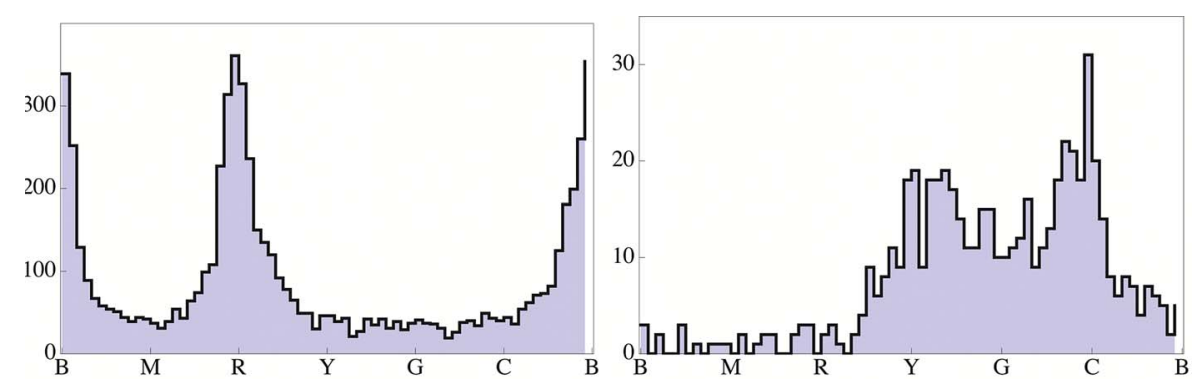

Fig. 13. (Color online) Density of hue angles for the simulation. At left the density for dark colors, at right the density for light colors. Notice that dark colors are predominantly red or blue and light colors predominantly yellow or cyan. 
no doubt find dominant signs of ecological causes of nonuniformity by selecting very specific scenes (both the Finnish flowers and the NCSU objects databases are apparently too general) or rigorously decreasing the sampling size. In the latter case one would merely have an undersampling artifact, but the former case is likely to be of interest in various niche environments.

Viewed from this perspective color vision "works" because, at least for vivid colors, there is an almost one-toone (give or take a little slack) relation between colors and reflectance spectra. (This is the basis of Ostwald's version of colorimetry [34,70]. A modern concept that is closely related is Wyszecki's Hypothesis [71].) This is due to the statistics of natural reflectance spectra, especially the fact that the spectral articulation tends to be very limited. Without this constraint, metamerism would destroy the (almost) one-to-one relation and-even more importanthardly any vivid colors would occur at all. Thus color vision is indeed well tuned to the physics of the human biotope. The spectral tuning of the visual system is best summed up by the fact that it is close to a coarse-grained spectroscope with only three spectral bins in the spectral range 426-633 $\mathrm{nm}$ (5\%-95\% quantiles), the divisions being at about 483 and $567 \mathrm{~nm}$ (at least for common daylight illumination). It makes sense to look for an ecological reason for that. Given this structure, the accumulations seen in the prior statistics of object colors are determined by the resulting edge color loci.

In this paper only bare-bones colorimetry was used (no eye measure-e.g., Munsell structure-used at all); thus only the input to the brain is considered. The physiology ends with the photon catches of the retinal pigments, and psychology enters only in the "judgment of equality," which may be interpreted as "judgment of equal input to the brain," which really does not involve "judgment" in the conventional sense. No brain processes proper nor experiental qualities (qualia) are invoked at any stage of the argument. Thus the arguments are independent of whether one prefers "opponent-color" descriptions [72-74], and so forth [75].

The non-uniform statistics of object colors is an immediate consequence of certain colorimetric structures that have been known for ages (on the time scale of modern science) but are generally disregarded in modern colorimetric praxis. Thus "edge colors" were described by Goethe [38] at the end of the 18th century, the tripartition of the spectrum as a preferred basis of primaries is due to Schopenhauer $[45,46]$ at the early part of the 19 th century, the "Principle of Inner Symmetry" is due to Ostwald [59-61] at the beginning of the 20th century, and the "optimal colors" and "color solid" are due to Schrödinger's [33] paper of 1920. These structures are all that is needed to derive the non-uniform statistics of object colors. Although the numerical simulations in this paper use modern CIE data, the conclusions do not depend on that. One could have used Maxwell's data (of the mid 19th century) with essentially the same results.

It is perhaps of some interest that the structure of the space of object colors is largely dominated by the geometry of the edge color locus. Although rarely acknowledged in the colorimetric literature, the edge colors [38] (German: Kantenfarben) are really the natural basis for the object colors, moreover; they are (after white and black) the most frequent colors. In the outdoors environment the long-wavelength-pass edge colors are familiar from the frequent occurrence of red, orange, yellow, and white flower colors.

\section{ACKNOWLEDGMENTS}

This work was done while I was visiting the ENS/INRIA projectteam WILLOW as an invited professor with the Département d'Informatique of École Normale Supérieure. I thank Jean Ponce for his hospitality.

\section{REFERENCES AND NOTES}

1. T.-W. Lee, T. T. Wachtler, and T. J. Sejnowski, "Color opponency is an efficient representation of spectral properties in natural scenes," Vision Res. 42, 2095-2103 (2002).

2. A. Lewis and L. Zhaoping, "Are cone sensitivities determined by natural color statistics?" J. Vision 6, 285-302 (2006).

3. D. T. Lindsey and A. M. Brown, "Sunlight and 'Blue'," Psychol. Sci. 15, 291-294 (2004).

4. G. Wyszecki and W. S. Stiles, Color Science (Wiley, 1967).

5. The problem is that the experimental uncertainly is usually fixed in absolute terms (for typical databases of the order of 0.01), whereas for reflectance factors near zero or one it is the difference from zero or one that is relevant, and this difference easily drowns in the experimental uncertainty.

6. F. Long and D. Purves, "Natural scene statistics as the universal basis of color context effects," Proc. Natl. Acad. Sci. U.S.A. 100, 15190-15193 (2003).

7. F. Long, Z. Yang, and D. Purves, "Spectral statistics in natural scenes predict hue, saturation, and brightness," Proc. Natl. Acad. Sci. U.S.A. 103, 6013-6018 (2006).

8. D. L. Philipona and J. K. O'Regan, "Color naming, unique hues, and hue cancellation predicted from singularities in reflection properties," Visual Neurosci. 23, 331-339 (2006).

9. D. B. Judd and G. Wyszecki, Color in Business, Science, and Industry (Wiley, 1975).

10. J. C. Maxwell, "On the theory of compound colours, and the relations of the colours of the spectrum," Philos. Trans. R. Soc. London 150, 57-84 (1860).

11. M. G. J. Minnaert, Light and Color in the Outdoors (Springer-Verlag, 1993).

12. D. Katz, Die Erscheinungsweisen der Farben und ihre Beeinflussung durch die individuelle Erfahrung (Barth, 1911).

13. P. Bouguer, L'Essai d'Optique sur la Gradation de la Lumière (Gauthier-Villars et Cie, 1729).

14. J. H. Lambert, Photometria sive de mensure de gratibus luminis, colorum umbræ (Eberhard Klett, 1760).

15. A "reflectance factor" is the ratio of the radiance scattered to the eye by some surface and the radiance that would have been scattered to the eye by an ideal Lambertian surface. The advantage of the "reflectance factor" is that this avoids complications with the viewing and illumination geometries.

16. F. Nicodemus, "Directional reflectance and emissivity of an opaque surface," Appl. Opt. 4, 767-773 (1965).

17. M. Born and E. Wolf, Principles of Optics: Electromagnetic Theory of Propagation, Interference and Diffraction of Light (Cambridge Univ. Press, 1999).

18. G. Kortüm, Reflexionsspektroskopie (Springer, 1969).

19. L. T. Maloney, "Evaluation of linear models of surface spectral reflectance with small number of parameters," J. Opt. Soc. Am. A 3, 1973-1683 (1986). 
20. H. S. Fairman and M. Brill, "The principal components of reflectances," Color Res. Appl. 29, 104-110 (2004).

21. J. A. Worthey and M. Brill, "Principal components applied to modeling: dealing with the mean vector," Color Res. Appl. 29, 261-266 (2004)

22. A. Schuster, "Radiation through a foggy atmosphere," Astrophys. J. 21, 1-22 (1905).

23. P. Kubelka and F. Munk, "Ein Beitrag zur Optik der Farbanstriche," Z. Tech. Phys. 12, 92-99 (1931).

24. H. Jeffreys, Scientific Inference (Cambridge Univ. Press, 2003).

25. E. T. Jaynes, Probability Theory: The Logic of Science (Cambridge Univ. Press, 1931).

26. A. V. Oppenheim, R. W. Shafer, and T. G. Stockham, Jr., "Nonlinear filtering of multiplied and convolved signals," Proc. IEEE 56, 1264-1291 (1968).

27. D. Sen, M. N. S. Swamy, and M. O. Ahmad, "Unbiased homomorphic system and its application in reducing multiplicative noise," IEEE Proc. Vis. Image Signal Process. 153, 521-537 (2006).

28. E. L. Krinov, "Spectral'naye otrzhatel'naya sposobnost' prirodnykh obrazovanii," Izd. Akad. Nauk USSR, emphSpectral reflectance properties of natural formations, G. Belkov, transl., TT-439 (National Research Council of Canada, 1953).

29. O. Kohonen, J. Parkkinen, and T. Jääskeläinen, "Databases for spectral color science," Color Res. Appl. 31, 381-388 (2006).

30. Here the arc length of the short-wavelength-pass edge colors is used to define the quartiles and median of the wavelength domain. The value of the relative bandwidth quoted here is the interquartile range divided by the median. The visual band is thus extremely narrow by physical standards, and one has no reason to expect significant variations due to the physics.

31. CIE Proceedings Vienna Session 1963, Vol. B, 209-220(Committee Report E-1.4.1), (Bureau Central de la CIE, Paris, 1964).

32. R. S. Berns, Billmeyer and Saltzman's Principles of Color Technology (Wiley, 2000).

33. E. Schrödinger, "Theorie der Pigmente von größter Leuchtkraft," Ann. Phys. 62, 603-622 (1920).

34. P. J. Bouma, Physical Aspect of Colour (Macmillan, 1971).

35. Consider the locus of points $\left\{x_{1}, x_{2}, \ldots, x_{n}\right\} \in \mathbb{R}^{2}$ under the constraint $0 \leqslant x_{i} \leqslant a_{i}$. For $n=1$, one has the segment [0, $\left.a_{1}\right]$, for $n=2$ the rectangle with vertices $\{0,0\},\left\{a_{1}, 0\right\},\left\{a_{1}, a_{2}\right\}$ and $\left\{0, a_{2}\right\}$, i.e., a rectangle, and so forth. In general, one obtains a "cuboid" (for $a_{1}, \ldots, a_{n}=1$ a unit hypercube) which is-in the absence of a metric-more appropriately called a "hyperparallelepiped."

36. S. Axler, Linear Algebra Done Right (Springer, 1997).

37. A. Kirschmann, "Das Umgekehrte Spektrum Seine Komplementarverhaltnisse," Phys. Z. 18, 195-205 (1917).

38. J. W. von Goethe, Zur Farbenlehre (Cotta, 1808-1810).

39. I. Newton, Opticks or a Treatise on the Reflexions, Refractions, Inflexions and Colours of Light, 4th ed. (William Innys, 1730).

40. The volume of the color solid is only 1.43 times that of the inscribed parallelopiped of maximum volume, whereas the circumscribed sphere of a cube has 2.72 (namely, $\pi \sqrt{3} / 2$ ) times the volume of that cube. (Such numbers are very relevant yet cannot be found in textbooks on colorimetry.) Thus the color solid is rather close to the linear transform of a unit cube.

41. W. Blaschke, Vorlesungen der Differentialgeometrie und geometrische Grundlagen von Einsteins Relativittstheorie. II. Affine Differentialgeometrie, bearbeitet von K. Reidemeister. Erste und zweite Auflage (Julius Springer, 1923).

42. D. Davis, "Generic affine differential geometry of curves in $\mathbf{R}^{\mathrm{n}}$," Proc. R. Soc. Edinburgh, Sect. A: Math. Phys. Sci. 136, 1195-1205 (2006).

43. J. B. Cohen and W. E. Kappauf, "Metameric color stimuli, fundamental metamers, and Wyszeckis metameric blacks," Am. J. Psychol. 95, 537-564 (1982).

44. Cohen implicitly applies the usual Cartesian inner product on radiant power spectra on wavelength basis. This turns the space of spectra into a Hilbert space. The visual projection then can be turned into an orthogonal projection, thus enabling the definition of "visual space." Accepting an orthonormal basis for visual space then leads to a representation very similar to the one constructed here. However, this seems more of a lucky strike. Moreover, the metric adopted by Cohen is rather ad hoc and hard to defend. It is certainly preferable to avoid the choice of metric altogether.

45. A. Schopenhauer, Über das Sehn und die Farben (Johann Friedrich Hartknoch, 1816).

46. R. A. Crone, "Schopenhauer on vision and the colors," Doc. Ophthalmol. 93, 61-71 (1997).

47. H. S. M. Coxeter, Introduction to Geometry, 2nd ed. (Wiley, 1989).

48. The volume of the color solid is 1.43 times the volume of the maximum volume crate. The cube root of 1.43 is 1.13 , which yields a more intuitive (because linear instead of volumetric) measure of the difference.

49. R. G. Kuehni, "Focal color variability and unique hue stimulus variability,” J. Cogn. Culture 5, 409-426 (2005).

50. W. A. Thornton, "Luminosity and color-rendering capability of white light,” J. Opt. Soc. Am. 61, 1155-1163 (1991).

51. The dominant wavelengths of the secondary colors are $483 \mathrm{~nm}$ for cyan and $567 \mathrm{~nm}$ for yellow. (Magenta is complementary to green and might-formally—be said to have dominant wavelength $-527 \mathrm{~nm}$.)

52. S. Skiena, "Hasse diagrams," in Implementing Discrete Mathematics: Combinatorics and Graph Theory with Mathematica (Addison-Wesley, 1990), pp. 163, 169-170, and 20-208.

53. G. Birkhoff, Lattice Theory, 3rd ed., Colloquium Publications 25 (American Mathematical Society, 1967).

54. B. S. W. Schröder, Ordered Sets: An Introduction (Birkhäuser, 2003).

55. J. D. Foley, A. van Dam, S. K. Feiner, J. F. Hughes, Computer Graphics: Principles and Practice in C (AddisonWesley Professional, 1995).

56. A. F. Moebius, Der baryzentrische Calcul (orig. 1827) (Georg Olms, 1976).

57. Ratios of areas are not invariant in the chromaticity diagram. Almost all representations in the textbooks give an extremely lopsided view of the gamut of object colors. The boundary of the gamut (that is the spectrum locus) is the image of the black point and is irrelevant for most applications, despite the fact that it is usually suggested that it is the locus of the most vivid colors (apparently because it is monochromatic). But the spectral reflectances of monochromatic object colors are blip functions of vanishing support, thus these colors are blacks!

58. Of the NCSU objects data base only 3 out of the 170 samples yield more than $1 \%$ over- or underflow, the worst case being a $3 \%$ underflow in the green channel, which is perceptually irrelevant.

59. W. Ostwald, Farbkunde (S. Hirzel, 1923).

60. W. Ostwald, Die Farbenfibel (Unesma, 1926)

61. W. Ostwald, Er und Ich (Theodor Martins Textilverlag, 1936).

62. W. Ostwald, Der Farbatlas (Unesma, 1917).

63. M. Richter, "Untersuchungen zur Aufstellung eines empfindungsgemäß gleichabständigen Farbsystems," Z. Wiss. Photographie 45, 139-162(1950).

64. Ph. O. Runge, Farbenkugel oder Construction des Verhältnisses aller Mischungen der Farben zu einander und ihrer vollständigen Affinität; mit angehängtem Versuch einer Ableitung der Harmonie in den Zusammenstellungen der Farben (Friedrich Perthes, 1810).

65. P. Klee, The Thinking Eye (Lund Humpries, 1961).

66. S. Quiller, Color Choices (Watson-Guptill Publications, 1989).

67. R. G. Kuehni, "Development of the idea of simple colors in the 16th and 17th centuries," Color Res. Appl. 32, 158-162 (2004).

68. A. H. Munsell, A Color Notation (G. H. Ellis Co., 1905). 
69. W. Blaschke, Vorlesungen über Differentialgeometrie und geometrische Grundlagen von Einstein's Relativiteitstheorie. Volume 1, Elementare Differentialgeometrie (Julius Springer, 1921).

70. S. Rösch, "Die Kennzeichnung der Farben," Phys. Z. 29, 83-91 (1928).

71. G. Wyszecki, "Valenzmetrische Undersuchung des Zusammenhanges zwischen normaler und anomaler Trichromasie," Farbe 2, 39-52 (1953).
72. E. Hering, "Zur Lehre vom Lichtsinn," Sitzungsber. K. Preuss. Akad. Wiss. Math. Naturwiss. Kl. 5-24 (1872).

73. L. M. Hurvich and D. Jameson, "An opponent-process theory of color vision," Psychol. Rev. 64, 384-404 (1957).

74. E. Schrödinger, "Über das Verhältnis der Vierfarben-zur Driefarbentheorie," Sitzungsber. Akad. Wiss. Wien Math. Naturwiss. Kl. Abt 2a 134, 471-490 (1925).

75. E. H. Land, "The retinex theory of colour vision," Proc. R. Inst. Great Britain 47, 23-58 (1974). 